\title{
Aportes de la recreación a la interpretación ambiental en las áreas naturales protegidas
}

Contributions of Recreation to Environmental

Interpretation in Protected Natural Areas

Contribuições da recreação para a interpretação ambiental em unidades de conservação

\author{
Astrid Bibiana Rodríguez Cortés \\ Liliana Maribel Mora González ${ }^{* *}$
}

Recibido: 20 abril del 2020

Aprobado: 23 de noviembre del 2020

https://doi.org/10.12804/revistas.urosario.edu.co/territorios/a.8958

Para citar este artículo

Rodríguez Cortés, A., \& Mora González, L. (2021). Aportes de la recreación a la interpretación ambiental en las áreas naturales protegidas. Territorios, (44-Especial), 129-123. https://doi.org/10.12804/revistas. urosario.edu.co/territorios/a.8958

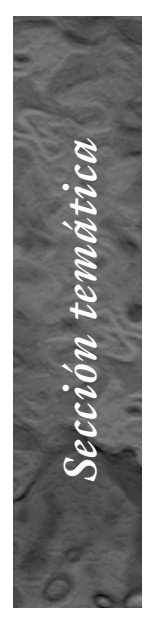

* Universidad Pedagógica Nacional. Correo electrónico:abrodriguez@ pedagogica.edu.co. ORCID: https://orcid.org/00000002-6023-2933

** Universidad Pedagógica Nacional. Correo electrónico:Immorag@ pedagogica.edu.co. ORCID: https://orcid.org/00000003-1090-2190 
Palabras clave

Interpretación ambiental; recreación; áreas protegidas; campo de acción; turismo.

Keywords

Environmental interpretation; recreation; protected areas; field of action; tourism.

Palavras-chave Interpretação ambiental; recreação; áreas protegidas; campo de ação; turismo.

\section{tersitarias 44 Especial}

\section{RESUMEN}

La interpretación ambiental sostiene una propuesta de educación basada en la experiencia, el interés, la construcción de significados y el reconocimiento de las potencialidades del entorno para el desarrollo de actividades con una finalidad educativa en espacios abiertos. Este artículo presenta una reflexión sobre ciertas actividades de interpretación ambiental propuestas por estudiantes de Licenciatura en Recreación y Turismo de la Universidad Pedagógica Nacional, implementadas en algunas áreas protegidas de Colombia. En las actividades propuestas se evidencia cómo la recreación aporta al ejercicio de interpretación ambiental, constituyéndose en un campo de acción desde el cual los licenciados pueden aportar a la conservación de la diversidad biofísica y sociocultural colombiana, así como reconocer las áreas protegidas como escenarios pedagógicos que promueven el cuidado, preservación y valor social de los entornos naturales. Estos elementos sugieren que la articulación entre la interpretación ambiental y la recreación desde el saber pedagógico configuran un escenario de acción que favorece la relación de las áreas protegidas con distintos actores del territorio.

\section{ABSTRACT}

The environmental interpretation supports an education proposal based on experience, interest, and the construction of meanings and the recognition of the potential of the environment, for the development of activities with an educational purpose in outside spaces. This article shows a reflection about some environmental interpretation activities proposed by students of the Degree in Recreation and Tourism of the Universidad Pedagógica Nacional and implemented in some protected areas of Colombia. The proposed activities show how recreation contributes to the exercise of environmental interpretation, constituting a field of action from which graduates can contribute to the conservation of Colombian biophysical and sociocultural diversity, as well as recognizing protected areas as pedagogical scenarios that promote the care, preservation and social value of natural environments. These elements suggest that the articulation between environmental interpretation and recreation from the pedagogical knowledge configure a scenario of action that help the relationship of protected areas with different actors of the territory.

\section{RESUMO}

A interpretação ambiental sustenta uma proposta educacional baseada na experiência, no interesse, na construção de significados e no reconhecimento das potencialidades do meio ambiente, para o desenvolvimento de atividades com uma finalidade educativa em espaços abertos. Este artigo apresenta uma reflexão sobre algumas atividades de interpretação ambiental propostas por alunos de graduação do curso de licenciatura em Recreação e Turismo da Universidade Pedagógica Nacional e implementadas em algumas áreas protegidas da Colômbia. Nas atividades propostas, fica evidente como a recreação contribui para o exercício da interpretação ambiental, tornando-se um campo de ação a partir do qual os graduandos podem contribuir para a conservação da diversidade biofísica e sociocultural colombiana, bem como reconhecer as áreas protegidas como cenários pedagógicos que promovem o cuidado, preservação e valor social dos 
ambientes naturais. Esses elementos sugerem que a articulação entre a interpretação ambiental e a recreação a partir do saber pedagógico configuram um cenário de ação que favorece a relação das unidades de conservação com os diferentes atores do território.

\section{Introducción}

Desde finales de los años treinta, en Colombia se constituyeron los primeros acercamientos al Sistema Nacional de Áreas Protegidas (SINAP). Inicialmente, con este sistema se buscaba "conservar el recurso hídrico necesario para la industria cañera en el Valle del Cauca" (Rojas, 2014 , p. 157). Más adelante, con el movimiento ambientalista de los años sesenta y la asistencia de Colombia a la primera Conferencia Mundial sobre Medio Ambiente Humano realizada en Estocolmo, del 5 al 16 de junio de 1972, el país se comprometió internacionalmente con la creación, implementación de programas, planes e instituciones que preservaran el medio ambiente y la biodiversidad del país (Rodríguez, 2017).

En 1992 se firmó el Convenio de Biodiversidad, ratificado por la Ley 165 de 1994 que obliga a los firmantes a la creación del Sistema Nacional de Áreas Protegidas (Rojas, 2014). Esta decisión le permitió al país hacer conciencia de su biodiversidad cultural, natural y social. Si bien en un primer momento, como lo presenta Rojas (2014), el modelo de conservación que acogió Colombia estuvo basado en mantener la naturaleza alejada del ser humano con el fin de conservarla prístina, años más adelante se comprendió la necesidad de establecer una relación cercana entre el ser humano y la naturaleza con el fin de trabajar por su preservación. En la actualidad se reconoce a Colombia como uno de los países biodiversos del mundo, cuenta con 59 parques naturales que totalizan 12602320,7 hectáreas, lo que representa más del $12 \%$ del territorio nacional, correspondiendo el 9,98\% a territorio terrestre y el $1,3 \%$ a territorio marino (SINAP, 2014). Algunos de los objetivos que buscan las áreas protegidas tienen que ver con la conservación del patrimonio natural y cultural del país. En este sentido, sus estrategias para fomentar la gestión y conservación tienen que ver principalmente con en el apoyo en procesos educativos y turísticos.

Indudablemente la belleza de los Parques Naturales es atractiva para el ecoturismo, Colombia recientemente se ha declarado con un alta vocación para este tipo de turismo, si bien, desde las concepciones de la Organización Mundial del Turismo, esta actividad debe ocasionar el menor impacto posible en el ambiente, en realidad, estas áreas protegidas siguen reportando impactos negativos frente a la práctica turística. Ante esta situación se considera que una de las posibles acciones para contrarrestar dichos impactos tiene que ver con recurrir a fomentar procesos educativos de conservación, cuidado territarios 44 Especial 111 
y preservación en turistas y lugareños. Esta perspectiva pretende crear una conciencia sobre la necesidad de la apropiación del territorio como bien social y cultural.

Siguiendo lo planteado, la Licenciatura en Recreación concibe a los Parques Naturales principalmente como escenarios pedagógicos de alto valor social. Uno de los objetivos de la formación de licenciados en Recreación tiene que ver con diseñar estrategias didácticas que relacionen la educación, la recreación y la práctica turística. Aunque este ejercicio no ha sido nada fácil, una de las estrategias que más ha permitido enriquecer la práctica docente de los estudiantes tiene que ver con la interpretación ambiental. Este campo se ha concebido para algunos autores como una especialización de la Educación Ambiental, sin embargo, Ziperovich (2004) plantea otra perspectiva:

Con el auge del turismo orientado a la utilización de los parques surge el problema de que el turista no solo busca acumular datos académicos y cientificistas de folleto u orales sobre las especies, animales o vegetales de la zona, sino también vivenciar ese espacio, sentirse parte y disfrutar en forma activa del entorno; jugar a ser un miembro más del sistema, como verdaderamente somos.

Es así como, fundamentalmente en Estados Unidos de América, surge la necesidad de desarrollar determinadas actividades tendientes a darle participación al visitante de los parques. De esta manera surge una gama de propuestas que se han dado en llamar interpretación ambiental (p. 79).

Es así como desde los años cincuenta el escritor y periodista norteamericano, Freeman Tilden, fue uno de los primeros en establecer principios y teorías sobre la interpretación del patrimonio, consideraba la interpretación ambiental como "una actividad educativa que pretende revelar significados e interpretaciones a través del uso de objetos originales, por un contacto directo con el recurso o por medios ilustrativos en lugar de simplemente comunicar información literal" (Tilden, 1957, citado por Ham, 1992, p. 3). Sin embargo, el término se popularizó en los años setenta por los movimientos y cumbres ambientalistas. Entonces, la mirada sobre el patrimonio ambiental se articuló con perspectivas educativas alternativas que concebían la importancia de la educación en y para el tiempo libre, por ello, para Morales la interpretación ambiental consiste en "la revelación in situ del significado del patrimonio natural, cultural o histórico, al público general que visita ciertos lugares en su tiempo de ocio" (citado en Pellegrini et al., 2007, p. 607). En esta misma perspectiva, Ziperovich (2004) y Mayorga (2012) plantean que la interpretación ambiental se trata de conjugar la relación educación-recreación y turismo.

En ese marco, la propuesta que hace la Licenciatura en Recreación al retomar la interpretación ambiental como estrategia de enseñanza y aprendizaje, busca que 
los licenciados que están en formación, diseñen experiencias recreativas que se realizan en algunos Parques Naturales con los cuales la Licenciatura tiene convenio de prácticas pedagógicas. ${ }^{1}$ El diseño de estas experiencias tiene como objetivo que los turistas comprendan el valor del patrimonio natural y cultural, además de crear una interrelación de aprendizaje, disfrute e interés por el medio natural. De esta manera, el propósito de este artículo es exponer algunas experiencias pedagógicas de interpretación ambiental desarrolladas por los estudiantes de la Licenciatura en Recreación en algunos Parques Naturales de Colombia.

\section{La práctica pedagógica como una apuesta orientada a la conservación del territorio}

Antes de exponer las experiencias pedagógicas, es necesario reconocer las áreas protegidas como territorios en disputa. Aunque como áreas protegidas, el Convenio sobre la Diversidad Biológica (1992) se refiere a "un área geográficamente definida que está designada o regulada y gestionada para lograr específicos objetivos de conservación” (p. 3), reconociendo así una noción de territorio; la administración por parte del estado de las áreas protegidas - como estrategia de conservación a nivel internacional-, sus decisiones políticas (como el turismo), y el lugar de las poblaciones autóctonas que antaño se encontraban allí o llegaron posteriormente, asumen el territorio desde la tradición social configurando prácticas que pueden afectar los ecosistemas (agricultura o ganadería). Es decir, en las áreas protegidas confluyen distintas miradas sobre el territorio y su apropiación, que se encuentran desarticuladas y requieren apuestas educativas que permitan avanzar en la comprensión acerca de la importancia del cuidado y conservación de los ecosistemas en el país.

Las experiencias pedagógicas que se muestran a continuación, tienen su fundamento en la concepción de práctica educativa que ha venido construyendo la Licenciatura en Recreación de la Universidad Pedagógica Nacional. El programa académico la asume como

El espacio de reflexión-acción e investigación en torno a la integración, innovación, indagación y recontextualización de los saberes de orden cognitivo, ético, estético, pedagógico, didáctico y disciplinar en un contexto sociocultural especifico, que presenta necesidades e intereses diversos, en función de la labor y formación profesional del estudiante, su crecimiento personal y social (Universidad Pedagógica Nacional, 2013, p. 61).

Con esa orientación, la Licenciatura en Recreación está construyendo continuamente apuestas de intervención pedagógica en territorios que posibiliten la puesta en marcha del saber recreativo en función de lecturas de contexto integrales que
${ }^{1}$ La Universidad Pedagógica Nacional y Parques Nacionales Naturales vienen desarrollando acciones conjuntas desde hace más de 10 años. Recientemente se firmó el Convenio 001 de 2019, con una vigencia de 4 años, que tiene por objeto la "cooperación académica para el desarrollo de las prácticas académicas de formación profesional, realizando actividades que contribuyan a los procesos que se adelantan en Parques y permitiendo a los estudiantes de pregrado y postgrado de la universidad, aplicar y ampliar los conocimientos teóricos adquiridos en desarrollo del programa académico que cada uno se encuentre cursando" (s.p).

\section{territarias 44 Especial} 113 


\section{territorias} 44 Especial valoren las condiciones biofísicas y socioculturales del territorio. Las experiencias pedagógicas posibilitan a los licenciados en formación fortalecer los programas en las entidades que intervienen, desarrollar ejercicios de indagación e investigación y confrontar realidades territoriales. Con esta perspectiva se hace una valoración continua de sus acciones pedagógicas y se complementa su formación académica.

Los Parques Nacionales tienen una misión compleja como lo es la conservación, para ello, requieren la confluencia de diversas disciplinas que permitan gestionar y garantizar la protección de las áreas naturales. La conservación de dichas áreas está ligada principalmente a la educación de las poblaciones autóctonas, quienes habitan en estos territorios; pero a su vez, se necesita un despliegue de formación importante para los visitantes que realizan turismo en dichos lugares.

A lo largo de la historia, los Parques Nacionales han explorado diferentes apuestas educativas para sensibilizar a la población sobre la existencia e importancia de las áreas protegidas para la calidad de vida de las personas. En la presentación de esta experiencia, se destacan las intervenciones pedagógicas realizadas en áreas protegidas como el Parque Nacional Natural Sumapaz y el Parque Natural Nacional Cueva de los Guácharos, estás prácticas fueron orientadas a promover la conservación de las áreas protegidas con experiencias pedagógicas alternativas e incluyentes.

\section{Aplicaciones de la interpretación ambiental en la Licenciatura en Recreación}

A continuación, se señalan algunas propuestas construidas por los estudiantes en el marco de una alianza desarrollada entre la entidad Parques Nacionales Naturales de Colombia (PNN) y el programa académico de Licenciatura en Recreación de la Universidad Pedagógica Nacional, en específico, para el espacio académico Práctica Pedagógica en Turismo. En este sentido, para el programa académico, la práctica pedagógica:

\begin{abstract}
es un espacio de confrontación, proposición y acercamiento constante al hecho educativo, concibiendo este último no solo en la educación formal sino en los diversos contextos donde se produce. Por tanto, es misión del estudiante posicionar el saber recreativo y turístico en los diversos contextos de su acción docente, con perspectivas lúdicas y fortaleciendo el ocio como dimensión humana (Universidad Pedagógica Nacional, 2013, p. 62).
\end{abstract}

En dicho contexto, los estudiantes desarrollaron algunas propuestas pedagógicas de intervención en los territorios donde están ubicadas las áreas protegidas, con el propósito de aportar a la conservación de la biodiversidad biofísica y sociocultural de la región, a partir de sus conocimientos sobre recreación, turismo y saber pedagógico. 
El desarrollo de las áreas protegidas en Colombia ha respondido a acuerdos internacionales en relación con la conservación de la biodiversidad, pero también ha configurado una historia particular de la mano con las comunidades locales que viven al interior de las áreas protegidas, con las personas que se encuentran en las zonas aledañas y demás actores interesados en los territorios. Lo anterior, ha permitido que históricamente la gestión de las áreas protegidas acumule una experiencia de trabajo entre diversos actores que vinculan estrategias educativas encaminadas a favorecer la conservación de la diversidad biofísica, en primera instancia, y por consecuencia, a las comunidades en sí.

Con este panorama, en Colombia el aumento del turismo en las áreas protegidas ha sido importante en los últimos tiempos, pues antes, estos escenarios estaban restringidos por situaciones de orden público debido al conflicto armado. No obstante, ello también ha desencadenado múltiples problemas e impactos ambientales, en los cuales se hace necesario generar e implementar estrategias pedagógicas orientadas al ordenamiento de las actividades que se realizan en las áreas protegidas.

\section{Lazos intergeneracionales para la apropiación de las especies nativas del Parque Nacional Natural Sumapaz ${ }^{2}$}

\section{El Parque Nacional Natural Sumapaz es} un área protegida creada en 1977, cuenta con una extensión de 223179 hectáreas que se distribuyen altitudinalmente entre 700 m.s.n.m. y 4375 m.s.n.m. albergando ecosistemas de páramo y bosques andinos. Se encuentra ubicado entre los departamentos de Huila, Meta y Cundinamarca, a $23 \mathrm{~km}$ del casco urbano de la ciudad de Bogotá (véase figura 1). Sumapaz se considera un área de importancia hídrica para las cuencas del río Magdalena, el río Meta y el río Guaviare, aportando el agua hacia las regiones andina, orinocense y amazónica en nuestro país (PNN, 2019a).

Los campesinos que tradicionalmente viven al interior del Parque, han desarrollado de manera histórica actividades relacionadas con agricultura y ganadería; pero una vez Sumapaz fue declarado como área protegida, quedaron terminantemente prohibidas desde la legislación, conduciendo a tensiones en el territorio, lo cual ha obligado a las partes a generar acuerdos de manejo entre las comunidades y Parques Naturales Nacionales. Aunque Sumapaz es un área protegida cerrada al público:

el proceso de paz en Colombia ha facilitado la visita a los Parques Nacionales con vocación ecoturística que antes eran difíciles de visitar, como se refleja en el aumento de visitantes del 14,3\% entre 2016 y 2017 , alcanzando 1653000 de visitantes en el 2017, y del 16,2\% en el primer semestre de 2018 (PNN, 2019a, párr. 2).

Si bien Sumapaz no tiene vocación ecoturística, sí presenta en la actualidad una afluencia masiva de visitantes que
2 Propuesta elaborada para el espacio académico Práctica Pedagógica en Turismo, durante el 2018 por la estudiante Lida Ginneth Rojas Poveda, del programa académico $\mathrm{Li}$ cenciatura en Recreación y Turismo de la Universidad Pedagógica Nacional.

\section{territarios} 44 Especial 115 


\section{territorias} 44 Especial

Figura 1. Mapa de ubicación geográfica del Parque Nacional Natural Sumapaz
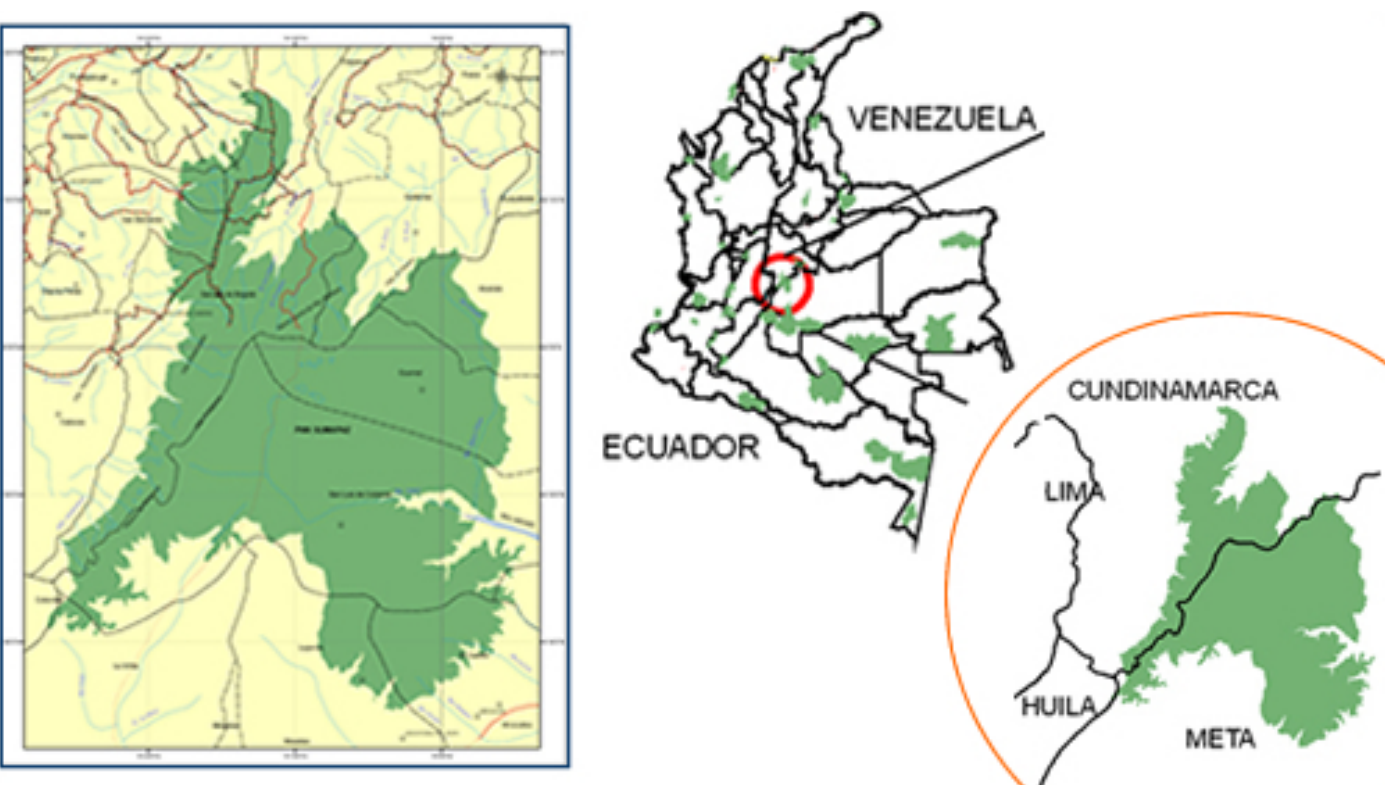

Fuente: Corporación Colombia Informa (2011).

ingresan al área protegida, sobre todo los fines de semana.

La falta de conocimiento de algunos visitantes sobre el significado de un área protegida, las actividades relacionadas con senderismo, fogatas, deportes como el bicimontañismo, deportes extremos como cuatrimotos, la realización de recorridos turísticos sin autorización del área protegida, entre otros, ha generado afectaciones sobre los ecosistemas que antaño eran inexplorados y que indudablemente plantean un desafío para los Parques Nacionales y para la región. Ante esta situación, la propuesta Lazos intergeneracionales para la apropiación de las especies nativas del Parque Nacional
Natural Sumapaz, planteó la posibilidad de reconocer especies nativas de Sumapaz a partir de una iniciativa que promoviera el diálogo de saberes, como una estrategia para la construcción colectiva del saber y la intersubjetividad, fortaleciendo las relaciones intergeneracionales y la producción de conocimientos, actitudes y prácticas en favor de la conservación de la biodiversidad.

Dicha propuesta fue implementada en tres momentos: la primera fase fue de recolección de información, por un lado, sobre las especies de fauna y flora emblemáticas del Parque Nacional Natural Sumapaz a partir de los diarios de campo que se registraban desde los diálogos 
con los funcionarios del Parque, y, por otro lado, datos sobre los visitantes que ingresaban los fines de semana al sector conocido como Laguna de Chisacá. Posteriormente, la segunda fase, plantea una actividad para promover el diálogo intergeneracional entre los visitantes al parque con relación a las especies de fauna y flora de Sumapaz. Por último, la tercera fase se constituyó en un momento de reflexión entre los estudiantes y funcionarios del Parque a partir del ejercicio adelantado con los visitantes.

En la fase inicial de diagnóstico, se encontró que el lugar era visitado por familias, estudiantes, ciclistas y motociclistas, cada grupo con intereses particulares como un día de paseo, motivos académicos, deporte y turismo. A partir de esa información, ${ }^{3}$ como estrategia de interpretación ambiental, se diseñó una caja negra cerrada en la cual apenas cabía una mano, en el interior se depositaban con anterioridad elementos del entorno, por ejemplo, una roca, una hoja, un residuo, una esponja de cocina (para explicar la función de los páramos), entre otros. La actividad consistía en ubicar a los grupos que llegaban al sector, saludarlos e invitarlos a que introdujeran su mano, uno a uno, en la caja negra e intentaran decir algo sobre lo que percibían allí, tal como se puede observar en la figura 2 .

La estudiante que lideraba la implementación de la actividad, Gineth Rojas, comenta en unos de sus diarios de campo:

[...] al inicio los niños hablan desde su emocionalidad con comentarios como: ¡ay la oreja de un conejo! ¡Súper como un algodón! ¡Eso rasguña! Se hacen notar, luego los jóvenes adultos que en ocasiones presumen de saber más, deslumbran con un ¡son matas! Que ubican a las plantas en un lugar con menor relevancia, aunque algunos de los jóvenes son conscientes en tanto llevan consigo un discurso de cuidado y protección, por otro lado, los adultos mayores están permeados por toda su experiencia y aunque si es transcendente, parece que cayeran en el discurso del iyo me lo sé! Y con orgullo cuentan y recuentan
Figura 2. Experiencia Sumapaz
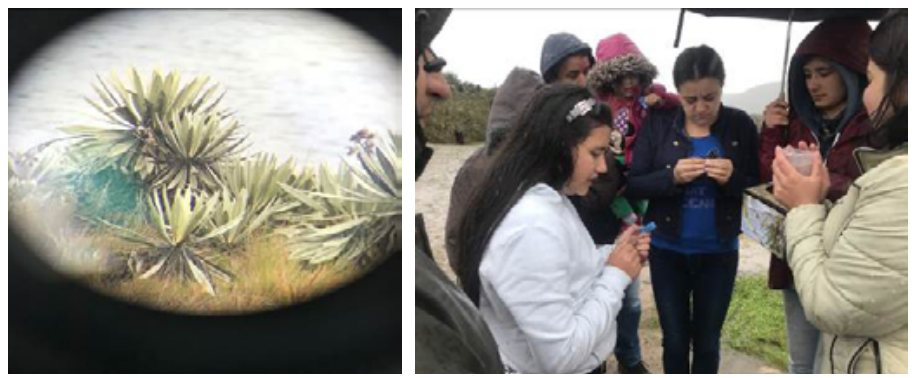

Fuente: Rojas (2018)

APORTES DE LA RECREACIÓN A LA INTERPRETACIÓN AMBIENTAL EN LAS ÁREAS NATURALES PROTEGIDAS
${ }^{3}$ Formato control de ingreso visitantes Parque Nacional Natural Sumapaz.

territarias 44 Especial 
4 Propuesta elaborada para el espacio académico Práctica Pedagógica en Turismo durante el 2018 por la estudiante Gabriela Natalia Barrera Martínez, del programa académico Licenciatura en Recreación y Turismo de la Universidad Pedagógica Nacional.

\section{territorias 44 Especial} 118 historias que giran a través del escenario, los que no conocían el páramo, la emoción vuelve a jugar un papel especial, junto con los niños se intercambian emociones, sensaciones y comentarios (p. 15).

El ejercicio adelantado con diferentes grupos de visitantes permitió una interacción diferente entre estos y el escenario al cual estaban ingresando. Esta experiencia les permitió acercarse al ecosistema de páramo de una forma distinta, reconociendo desde el tacto y la emoción la gran riqueza biofísica del lugar y generar actitudes en favor de su conservación.

De igual manera, la utilización de la caja para poner distintos elementos permitió explorar varias posibilidades (como la esponja de cocina) generando emociones, pensamientos y diálogos de diversa índole, en las cuales fue posible que personas mayores, adultos, jóvenes y niños hablaran desde sus saberes y experiencias. Para el caso de los niños, su conocimiento está mediado por la emoción que genera el lugar, en el caso de los adultos y personas mayores, está marcado por la experiencia y la memoria generando procesos de enseñanza-aprendizaje alrededor del cuidado de la vida. Así, se resalta cómo esta experiencia permitió compartir un aprendizaje en familia, una transmisión de saberes de forma intergeneracional donde "los beneficios actúan en doble dirección: los jóvenes se benefician de la mayor experiencia y perspicacia de los mayores y estos sienten que estan ayudando a otros con su cooperación y participación, y además se ven desde un ángulo más positivo las dos generaciones" (Osorio, 2002, p. 172).

\section{Actividades recreativas como medio para fortalecer la interpretación ambiental del Parque Nacional Natural Cueva de los Guácharos ${ }^{4}$}

El Parque Nacional Natural Cueva de los Guácharos fue la primer área protegida declarada en Colombia en 1960, tiene una extensión de 7134 hectáreas y está a una altura entre los 1630 m.s.n.m. y 2850 m.s.n.m. Se ubica entre los departamentos de Caquetá y Huila, y debe su nombre al ave que la representa denominada Guácharo (véase figura 3). Este Parque alberga ecosistemas de bosque andino y páramo, que permiten la conectividad ecológica entre la región Andina y la región Amazónica, es importante en la provisión de agua a las zonas de influencia y refugio de una alta biodiversidad (PNNb, 2019).

Esta área protegida no tiene habitantes en su interior. Sin embargo, en la zona aledaña viven campesinos e indígenas. Algunas organizaciones comunitarias prestan servicios de ecoturismo comunitario con los visitantes que llegan al Parque Nacional. Dicha iniciativa se enmarca en el programa Ecoturismo Comunitario promovido por Parques Nacionales en distintas regiones de Colombia, con el propósito de 
Figura 3. Ubicación geográfica del Parque Nacional Natural Cueva de los Guácharos

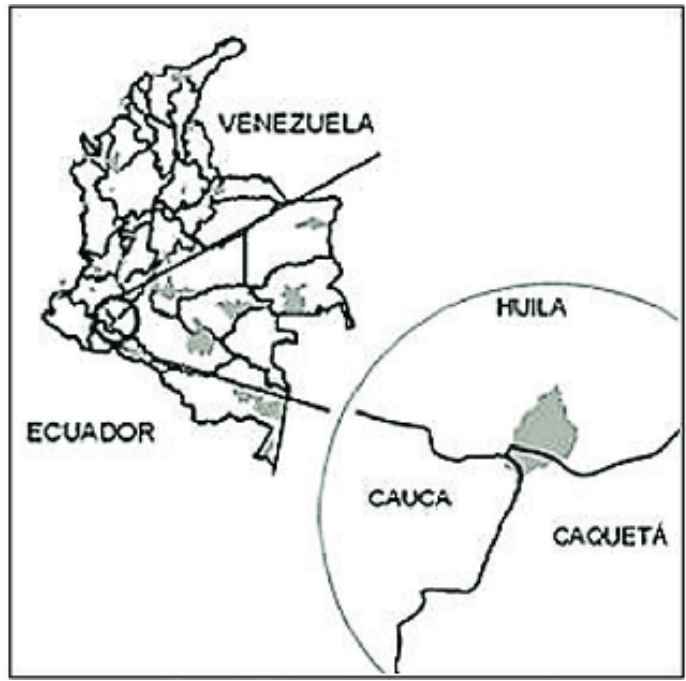

Fuente: Cifuentes et al. (2013).

establecer alianzas entre Parques y organizaciones comunitarias aledañas a las áreas del Sistema de Parques Nacionales Naturales, para la prestación de servicios y actividades ecoturísticas en áreas del Sistema de Parques Nacionales o su zona de influencia y el fortalecimiento de sus capacidades, mejorando la calidad de vida de las comunidades y la conservación de los recursos naturales (PNN, 2008).

Pese a que el Parque Nacional Natural Cueva de los Guácharos es un área protegida con vocación ecoturística y cuenta con un plan de interpretación ambiental, la implementación de este se ha visto limitada en tanto los guías comunitarios tienen una formación diversa y realizan los recorridos de una forma tradicional, que no tiene en cuenta los intereses de los grupos visitantes $y$, en ocasiones, se reduce a la mención de especies emblemáticas de la zona. Ante este escenario, la propuesta Actividades recreativas como medio para fortalecer la interpretación ambiental del Parque Nacional Natural Cueva de los Guácharos generada desde el programa académico de Licenciatura en Recreación, fue desarrollar actividades recreativas orientadas a fortalecer la interpretación ambiental para un sendero y consecuentemente generar conocimientos y actitudes en favor de la conservación del área protegida.

Esta propuesta se desarrolló en tres fases, en la primera se realizó un ejercicio de contextualización acerca de los 
documentos directrices del Parque y los ejercicios llevados en los recorridos realizados en el sendero sector Cedros; en la segunda se plantearon actividades recreativas basadas en juegos que permitieran a las personas integrarse, conocer datos básicos sobre el Parque y brindar orientaciones a los guías comunitarios y al equipo del Parque Nacional. Y en la tercera se realizó una valoración conjunta sobre los aprendizajes y los compromisos de conservación por parte de los visitantes. En los registros establecidos por la prácticante de Licenciatura en Recreación, Gabriela Natalia Barrera, comenta (2018):

[...] fue posible fortalecer la interpretación ambiental del Parque Nacional Natural Cueva de los Guácharos de una forma diferente, construyendo diferentes juegos y actividades para sus visitantes, en donde dependiendo de sus habilidades, su tiempo y disposición ahora se les ofrece diversas alternativas para aprender y reconocer la importancia del Parque y apoyar sus procesos de conservación a través de una forma lúdica (p. 18).

En la figura 4 se evidencia la implementación de la propuesta. El diálogo entre el equipo del Parque y los guías comunitarios fue fundamental para la construcción de un guión de inducción basado en el juego, que permitiera involucrar nuevas estrategias para acercar a los visitantes al conocimiento sobre el área protegida posibilitando, desde la interpretación ambiental, prevenir efectos negativos del turismo sobre los ecosistemas y generar capacidades en los guías y equipo del Parque. De la misma forma, la propuesta contribuyó al fortalecimiento de la alianza entre el Parque Nacional Natural Cueva de los Guácharos y organizaciones comunitarias de turismo como Corporación Andakíes y Fundación Cerca Viva, así como la dinamización de actividades en el centro de interpretación ambiental creando de manera conjunta lenguajes basados en la recreación como posibilidad para la transformación y el aprendizaje

Figura 4. Experiencia PNN Cueva de los Guácharos

\section{territarios 44 Especial}

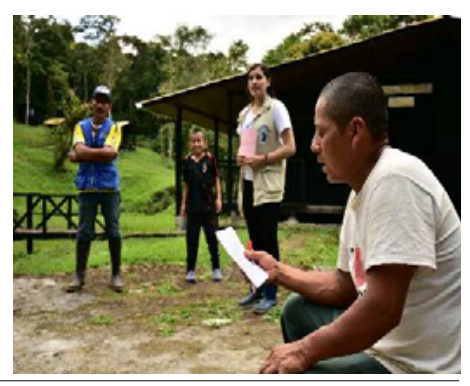

Fuente: Barrera (2018).
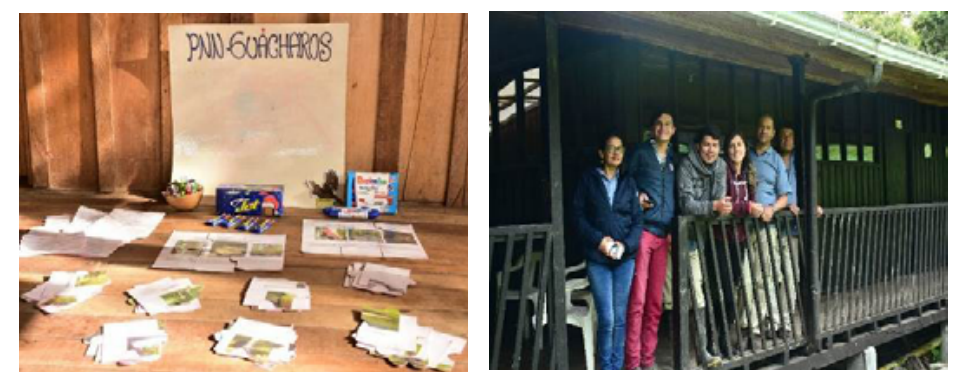

Astrid Bibiana Rodríguez Cortés, Liliana Maribel Mora GonZÁlez 
sobre la riqueza biofísica y sociocultural de nuestro país.

Las dos experiencias señaladas evidencian las áreas protegidas de Colombia como un escenario de valor incalculable para el desarrollo de actividades recreativas, una vez que en el momento histórico actual existe una afluencia turística sin precedentes. Las propuestas aportan elementos desde el saber pedagógico que piensan las características particulares de los visitantes, indagan acerca de su construcción de significados sobre el entorno, facilitando desde la vivencia, concientización acerca del uso y el valor de dichos escenarios, mitigando estrategias recreativas y afectaciones sobre los ecosistemas en la construcción de relaciones equilibradas entre los seres humanos y el medio en el que habitan.

La apuesta para la conservación de la diversidad biofísica y sociocultural en los territorios desde apuestas de interpretación ambiental elaboradas por licenciados en Recreación, ofrece diversas ventajas entre las cuales vale la pena destacar dos, la primera corresponde al enriquecimiento en la formación de licenciados que reconocen realidades territoriales complejas en las cuales confluyen distintos sectores y significados de apropiación, enfrentándose a la construcción de alternativas educativas dirigidas a distintos públicos en favor de la conservación y, la segunda, orientada a apoyar las estrategias de educación y comunicación generadas por Parques Nacionales lideradas por profesionales en pedagogía, quienes favorecen el fortalecimiento de capacidades de los equipos en las áreas protegidas y el reconocimiento acerca de la importancia de estas áreas para los seres humanos en todos los rincones del territorio nacional.

En este sentido, los licenciados en Recreación tienen un campo de acción importante en las áreas protegidas, en tanto su saber permite articular propuestas encaminadas hacia la conservación de la biodiversidad tomando como base las comunidades asentadas en las zonas y creando puentes comunicativos con los visitates. El saber del licenciado se hace necesario en tanto sus apuestas metodológicas comprenden particuaridades de los actores en el territorio y, permite construir diálogos que aportan a la gestión de las áreas desde acuerdos de manejo en todos los niveles que redunden en la construcción de conocimientos, actitudes y prácticas que favorezcan el cuidado y preservación de las áreas potegidas de Colombia.

\section{Reflexiones y desafíos}

Las mediaciones pedagógica e interpretativas desarrolladas por los estudiantes de la Licenciatura en Recreación en las áreas protegidas de Sumapaz y Cueva de los Guácharos, permitió a los visitantes acercarse a experiencias educativas a partir de sus propias vivencias, generando un ambiente de acercamiento, exploración y compenetración con la naturaleza, las territarios 44 Especial 121 


\section{territorias} 44 Especial expectativas de estos ejercicios interpretativos son la educación desde la experiencia, la reflexión en la acción y la sensibilización a partir de la comprensión de las necesidades y problemáticas del contexto.

La interpretación ambiental es una herramienta metodológica que permite a los licenciados en formación desarrollar competencias importantes en su quehacer, entre ellas: comprender el territorio como un espacio con características biofísicas donde se construyen símbolos y cultura que se materializa en la convivencia, las relaciones sociales y la participación ciudadana, aspectos preponderantes en la acción pedagógica. El diseño de acciones de interpretación ambiental según las necesidades de los territorios. Y, por último, generar reflexiones educativas que permitan la preservación y conservación de los ecosistemas.

Estos primeros acercamientos que realiza la Licenciatura en Recreación a la interpretación ambiental como un campo de estudio en desarrollo, plantean un gran desafío; hoy por hoy los procesos de conservación de la biodiversidad y su valoración deben llevarse de la mano con procesos educativos que sean creativos y posicionen el saber pedagógico en escenarios como las áreas protegidas. Colombia, de cara a los nuevos retos que le plantea la práctica ecoturística, debe proponer sinergias entre la academia y los ciudadanos que redunden en favor del cuidado, la conservación y la apropiación de sus territorios, propiciando el reconocimiento de la relación indisoluble entre ambiente, educación y recreación. Desde esta perspectiva, los responsables del turismo deben diseñar planes de acción que potencien la satisfacción de la experiencia turística sin que ello concurra en detrimento del ambiente, por ello, la interpretación ambiental es una herramienta fundamental para lograr conciencia social y ambiental.

\section{Referencias}

Barrera, G. (2018). Informe práctica pedagógica actividades recreativas como medio para fortalecer la interpretación ambiental del Parque Nacional Natural Cueva de los Guácharos. Universidad Pedagógica Nacional.

Cifuentes, E., Ramírez, M., León, J., \& Galvis, N. (2013). Dieta de los churucos colombianos (Lagothrix lagothricha lugens) en relación con la productividad de frutos en el Parque Nacional Natural Cueva de Los Guácharos Diet of Colombian Woolly Monkeys (Lagothrix lagothricha lugens) with Relation to the Productivity of Fruits in the PNN Cueva de Los Guácharos. Universidad de los Andes. https://www.researchgate. net/publication/320879302_Dieta_de_los_churucos_colombianos_Lagothrix_lagothricha_lugens_en_relacion_con_la_productividad_de_frutos_en_el_Parque_Nacional_Natural_Cueva_de_Los_ Guácharos_Diet_of_Colombian_Woolly_Monkeys_Lagothri 
Corporación Colombia Informa. (2011, 24 de marzo). Páramo de Sumapaz, en la mira de las hidroeléctricas. Colombia Informa. http://www.colombiainforma. info/paramo-de-sumapaz-en-la-mirade-las-hidroelectricas /

Ham, S. (1992). Interpretación ambiental: guia práctica. North American Press.

Osorio, E. (2002). Recreación y adulto mayor ocio para una vida plena. Funlibre.

Parques Nacionales Naturales de Colombia (PNN). (2008). Lineamientos para el ecoturismo comunitario en Colombia. PNN. https://www.parquesnacionales.gov.co/portal/wp-content/ uploads /2013/11/SSNA_Encuentro_Ecoturismo_2012.pdf

Parques Nacionales Naturales de Colombia (PNN). (2019a). Parque Nacional Natural Sumapaz. Parques Nacionales Naturales de Colombia. http://www. parquesnacionales.gov.co/portal/es/ parques-nacionales/parque-nacionalnatural-sumapaz/

Parques Nacionales Naturales de Colombia (PNN). (2019b). Parques Nacionales. Parques Nacionales Naturales de Colombia. https://www.parquesnacionales.gov.co/portal/es/ecoturismo/ region-andina/parque-nacional-natural-cueva-de-los-guacharos/

Pellegrini Blanco, N., \& Reyes Gil, R. (2007). Programa de interpretación ambiental en la Universidad Simón
Bolívar: sus recursos, su cultura y su historia. Educere, 11(39), 605611. http://ve.scielo.org/scielo. php? script $=$ sci_arttext\&pid $=$ S1316$49102007000400004 \& \operatorname{lng}=$ es \& tl $\mathrm{ng}=\mathrm{es}$

Rojas, L. (2018). Informe práctica pedagógica lazos intergeneracionales para la apropiación de las especies nativas del Parque Nacional Natural Sumapaz. Universidad Pedagógica Nacional.

Rodríguez, A. B. (2017). Subjetividades en el espacio público la ciclovía de la ciudad de Bogotá. Editorial Universidad de Cádiz; Universidad Pedagógica Nacional.

Rojas, Y. (2014). La historia de las áreas protegidas en Colombia, sus firmas de gobierno y las alternativas para la gobernanza. Sociedad y Economia, (27), 155-176. https://www.redalyc.org/ pdf/996/99631890007.pdf

SINAP. (2014). Sistema Nacional de Parques Naturales de Colombia. Parques Nacionales Naturales de Colombia. http://www.parquesnacionales.gov. co/portal/es/sistema-de-parquesnacionales-naturales.

Universidad Pedagógica Nacional (2013). Documento maestro programa Licenciatura en Recreación. UPN.

Ziperovich, A. (2004). Turismo y recreación. Trillas. 